\title{
Adaptive Algorithm of Edge Detection Based on Canny
}

\author{
Tao Ma, Jian-Dong Fang \\ Inner Mongolia University of Technology, College of Information Engineering, Hohhot, China \\ E-mail: matao2034@163.com, fangjd@163.com
}

\begin{abstract}
Aiming at the problem that the high and low thresholds need to be set artificially for Canny edge detection, this paper proposes an adaptive algorithm to set the high and low thresholds by the mean $\mathrm{S}$ and standard deviation $\delta$ of the non-maximally suppressed images. The simulation results show that the algorithm is feasible and effective, however, some pseudo-edges and edge discontinuities still exist in the processing of some images, so further improvement is needed.
\end{abstract}

Keywords-canny operator; edge detection; adaptive algorithm

\section{INTRODUCTION}

The edge of an image refers to a set of those pixels whose gray levels of surrounding pixels change abruptly, which is the most basic feature of the image. The basic idea of edge detection is to detect the edge points in the image and then connect the edge points into contours according to some strategy. Image edge detection is based on visual identification obstacle avoidance intelligent devices in a way to identify objects.

Two types of differential operators are often used in edge detection., one is the first-order, one is second-order. The first-order differential edge operator, also known as gradient edge operator, it makes use of the step of the image edge to detect the edge, that is, the image gradient is maximized at the edge. The second-order differential edge detection operator performs edge detection using the edge characteristic of the image,the step of the image at the edge results in a characteristic, which is that the value of the second-order differential at the edge of the image is zero. This method is also called the zero-crossing point operator and Laplacian operator. There are many kinds of operators for edge detection. Several commonly used operators ${ }^{[1]}$ include Roberts operator, Sobel operator, Prewitt operator and Canny edge detection operator.

Roberts operator is a local differential operator to find the edge, the detection of vertical edges is better than the oblique edge, high positioning accuracy, noise-sensitive, can not suppress the impact of noise, edge noise and less for image segmentation.

Prewitt operator and Sobel operator both have some restraining effect on noise. The weighted average is used, but the weight of Sobel operator varies with the distance.

Canny operator is not the edge detection by differential operator, but the edge detection optimization operator deduced under the condition of satisfying certain constraints.

Aiming at the problem that the high and low thresholds need to be set artificially for Canny edge detection, this paper proposes an adaptive algorithm to set the high and low thresholds by the mean $\mathrm{s}$ and standard deviation $\delta$ of the non-maximally suppressed images.

\section{CANNY EDGE DETECTION ALGORITHM}

Canny algorithm proposed three strict edge detection standards ${ }^{[2]}$ : (a) good signal to noise ratio; (b) high positioning accuracy; (c) single edge response. The basic idea of the Canny algorithm is to use the Gaussian function to smooth the image, then the gradient value and direction angle are obtained by convoluting the first order differential operator and the smoothed image, the final edge is then obtained by non-maximum suppression and dual threshold detection. Non maximum suppression not only can effectively restrain the multi response edge, but also can improve the positioning accuracy of the edge. Double-threshold detection can effectively reduce the leakage rate of the edge.

Edge Detection Based on Canny Operator ${ }^{[3]}$ :

1) De-noise: Usually using Gaussian filtering, which is a linear smoothing filter, suitable for the elimination of Gaussian noise, Gaussian filtering is essentially a weighted average of the entire image, the image of each pixel by its own value and the neighborhood of other pixels Value of the weighted average obtained. But in order to improve the efficiency of operation often use the filter template. In MATLAB, the function fspecial is used to calculate the filter template. The format is: $h=$ fspecial('gaussian', $N, \sigma$ ) .

Among them, $\mathrm{N}$ represents the order of the template,that is the size of the window, $\sigma$ is the standard deviation of the Gaussian function.

2) Calculate gradient values and direction angles: Assuming the gradient of the denoised image in the $x$ direction and $y$ direction is $M_{x}$ and $M_{y}$, where $M_{x}$ and $M_{y}$ are the convolution of Sobel template and image, then

The gradient values are:

$$
|\Delta f|=\sqrt{M_{x}^{2}+M_{y}^{2}}
$$

The gradient direction angle is:

$$
\theta=\arctan \left(M_{y} / M_{x}\right)
$$

Gradient direction angle $0^{\circ} \sim 360^{\circ}$ is merged into four 
directions $\theta^{\prime}: 0^{\circ}, 45^{\circ}, 90^{\circ}$ and $135^{\circ}$.

The rules are as follows:

The direction angles in the range $\left\lfloor-22.5^{\circ} \sim 22.5^{\circ}\right\rfloor$ and $\left[157.5^{\circ} \sim 202.5^{\circ}\right\rfloor$ are combined to the direction angle $0^{\circ}$;

The direction angles in the range $\left[22.5^{\circ} \sim 67.5^{\circ}\right\rfloor$ and $\left\lfloor 202.5^{\circ} \sim 247.5^{\circ}\right\rfloor$ are combined to the direction angle $45^{\circ}$;

The direction angles in the range $\left[67.5^{\circ} \sim 112.5^{\circ}\right\rfloor$ and $\left\lfloor 247.5^{\circ} \sim 292.5^{\circ}\right\rfloor$ are combined to the direction angle $90^{\circ}$;

The direction angles in the range $\left[112.5^{\circ} \sim 157.5^{\circ}\right\rfloor$ and $\left\lfloor 292.5^{\circ} \sim 337.5^{\circ}\right\rfloor$ are combined to the direction angle $135^{\circ}$.

\begin{tabular}{|l|l|l|}
\hline$(x-1, y-1)$ & $(x, y-1)$ & $(x+1, y-1)$ \\
\hline$(x-1, y)$ & $(x, y)$ & $(x+1, y)$ \\
\hline$(x-1, y+1)$ & $(x, y+1)$ & $(x+1, y+1)$ \\
\hline
\end{tabular}

Figure 1. The $3 \times 3$ neighborhood graph of pixel $(x, y)$.

3) Non-maximum suppression: $3 \times 3$ neighborhood is used as the template to detect, the pixels with the largest gradient value in the gradient direction are reserved as edge pixels, and the other pixels are deleted. As follows:

If $\theta^{\prime}(x, y)=0^{\circ}$, then check the pixels $(x-1, y),(x, y)$ and $(x+1, y)$;

If $\theta^{\prime}(x, y)=45^{\circ}$, then check the pixels $(x-1, y-1)$, $(x, y)$ and $(x+1, y+1)$;

If $\theta^{\prime}(x, y)=90^{\circ}$, then check the pixels $(x, y-1),(x, y)$ and $(x, y+1)$;

If $\theta^{\prime}(x, y)=135^{\circ}$, then check the pixels $(x-1, y+1)$, $(x, y)$ and $(x+1, y-1)$.

4) Dual threshold detection: Set two thresholds: a high threshold $t_{\text {high }}$ and a low threshold $t_{\text {low }}$. The detection process is:

- If the gradient value of the pixel $(x, y)$ is less than $t_{\text {low }}$, the pixel $(x, y)$ is a non-edge pixel;

- If the gradient value of the pixel $(x, y)$ is greater than $t_{\text {high }}$, the pixel $(x, y)$ is an edge pixel;

- If the gradient value of the pixel $(x, y)$ is between $t_{\text {high }}$ and $t_{\text {low }}$, it is necessary to further check the $3 \times 3$ neighborhood of the pixel, if the gradient of the pixel in the $3 \times 3$ neighborhood is greater than $t_{h i g h}$, then the pixel is the edge pixel; otherwise, the non-edge pixel .

\section{ADAPTIVE CANNY ALGORITHM}

In the traditional canny algorithm, the high and low thresholds are set manually and the threshold level can not automatically set according to the feature information of the image. Inappropriate high and low thresholds will affect the determination of the edge of the image. Pseudo-edge and edge discontinuity may occur. In terms of high and low threshold settings, A self-determined dual-threshold method based on gradient histogram and intraclass variance minimization has been proposed ${ }^{[4]}$. It has also been suggested that "the most value of the gradient pixel and the gradient value of the most value of the pixel double-threshold" approach ${ }^{[5]}$.

The method used in this paper is: first calculate the mean $\mathrm{s}$ and standard deviation $\delta$ of non maximum suppression image, then calculate the $t_{\text {high }}$ and $t_{\text {low }}$ by the formula $t_{\text {high }}=s+\rho_{1} \delta$ and $t_{\text {low }}=s-\rho_{2} \delta$.

\section{EXPERIMENTAL RESULTS AND ANALYSIS}

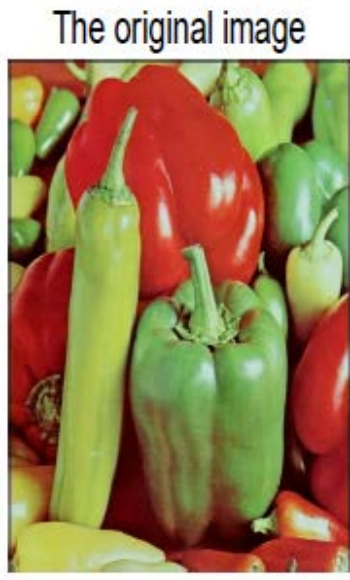

(a)

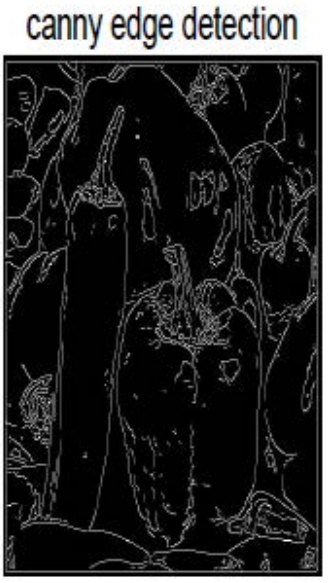

(b)
Figure 2 

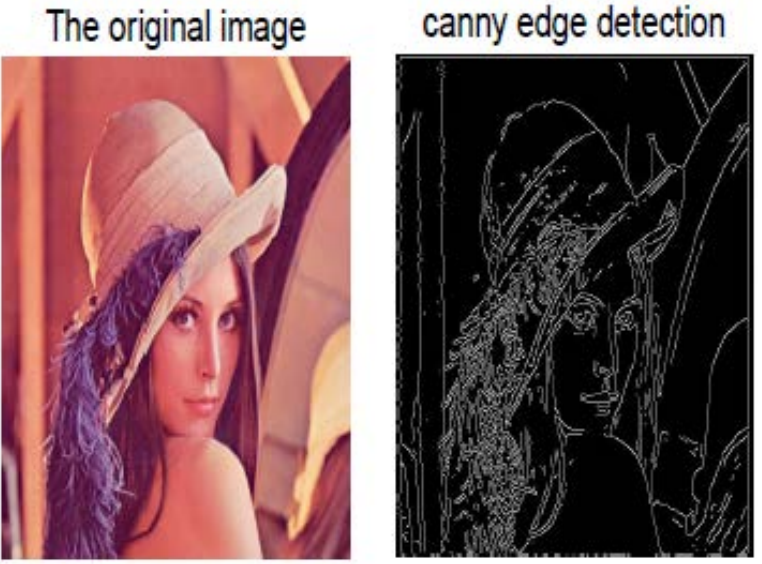

Figure 3
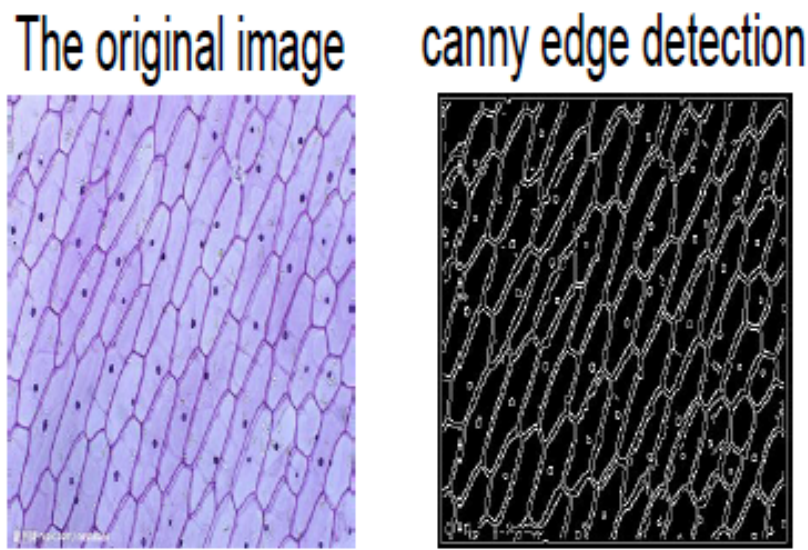

Figure 4
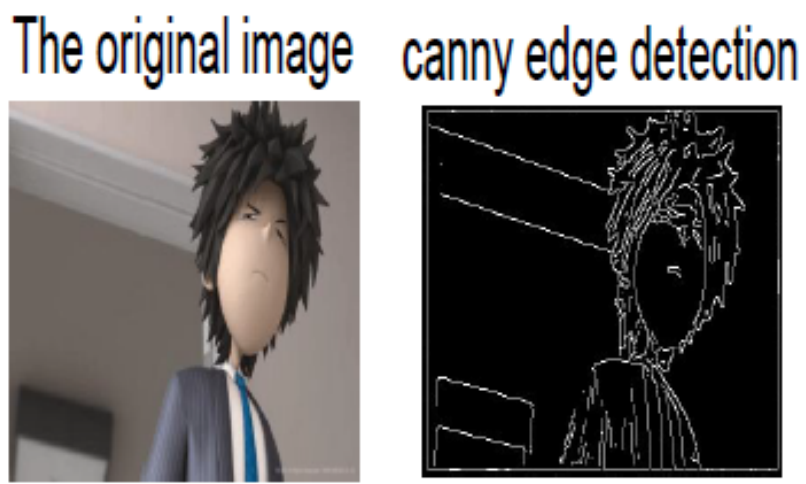

(a)

(b)

Figure 5.

Fig.2, Fig.3, Fig.4 and Fig.5 show that the edge of the image can be obtained effectively, and there are some pseudo-edge and edge discontinuities, which still need further improvement.

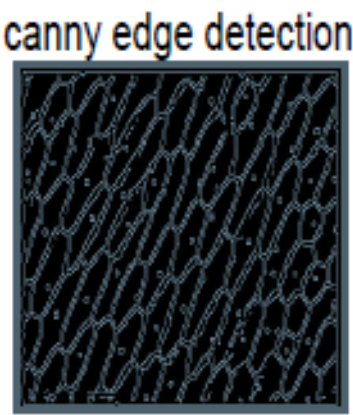

Fig.6

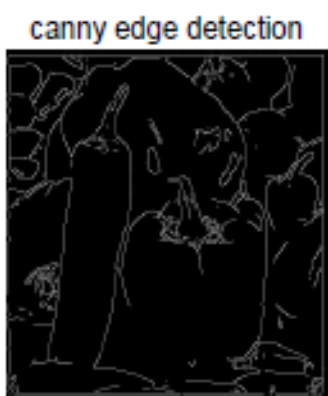

Fig. 8

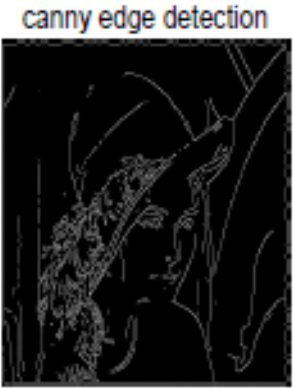

Fig.7
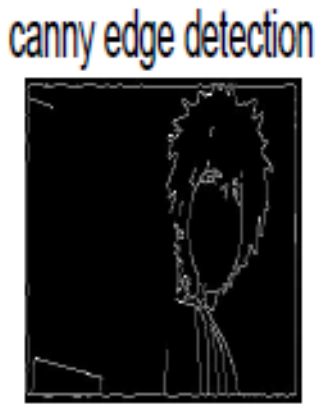

Fig.9
Fig.6, Fig.7, Fig.8, Fig.9 using the same fixed threshold for different images of the edge detection results; Fig.2 (b), Fig.3 (b), Fig.4 (b), Fig.5 (b) for the use of this method of edge detection results. The comparison shows that the method is feasible and effective, and can be applied to different image edge detection.

\section{CONCLUSION}

The experimental results show that using the mean $\mathrm{S}$ and the standard deviation $\delta$ of the non-maximally suppressed image, the feasibility and validity of setting the high and low thresholds can be realized. This method can avoid artificial interference and realize the threshold self-determination. The advantages of the canny algorithm are maintained, but it is found that there are still some false edges and edge discontinuities after processing some images, so further improvement is needed.

\section{ACKNOWLEDGMENTS}

This work is supported by Natural Science Foundation of Inner Mongolia Autonomous Region (No. 2014MS0619), and Inner Mongolia Science and Technology Project (No. 20120304). It's research findings that application system of intelligent decision making technology in agriculture and animal husbandry, by industry innovation talents team of Inner Mongolia Autonomous Region.

\section{REFERENCES}

[1] Duan Ruiling, Li Qingxiang, Li Yuhe. A Survey of Image Edge Detection Methods [J]. Optical Technology, 2005,03,pp.415-419. 


\section{ATLANTIS
PRESS}

Advances in Computer Science Research, (ACSR), volume 52

[2] Su Liancheng, Wang Dongwei. An Improved Canny Edge Detection Operator [J]. Journal of Yanshan University, 2012,05,pp.413-416.

[3] Zhao Xiaochuan. MATLAB image processing -- program implementation and modular simulation [M]. Beijing: Beijing University of Aeronautics and Astronautics Press, 2014,pp.176-178.
[4] Li Mu, Yan Jihong, Li Ge, Zhao Jie. Adaptive Canny operator edge detection technique [J] .Journal of Harbin Engineering University, 2007,09,pp.1002-1007.

[5] Wang Zhi, He Saixian. An adaptive edge detection method based on Canny theory [J] .Journal of Image and Graphics, 2004,08,pp.65-70. 